OPEN@ACCESS

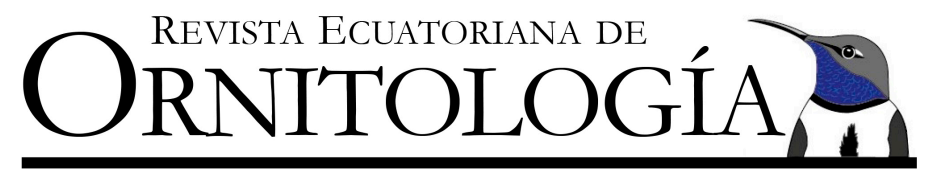

NOTAS DE CAMPO/FIELD NOTES

\title{
Vaquero Brilloso Molothrus bonariensis y Hornero Patipálido Furnarius leucopus: parasitismo de puesta
}

\author{
Ignacio Benjamín Navas Hojas \\ Pajareando Ando Ecuador \& Universidad de Guayaquil, \\ Av. Raúl Gómez Lince s/n y Av. Juan Tanca Marengo, Guayaquil, Ecuador \\ E-mail: benjamin.navas@hotmail.com \\ Editado por/Edited by: Harold F. Greeney \\ Recibido/Received: 02 Julio 2020 Aceptado/Accepted: 29 Mayo 2021 \\ Publicado en línea/Published online: 05 Julio 2021
}

El Vaquero Brilloso Molothrus bonariensis es una especie reconocida por ser parásito de puesta; es decir, que deposita sus huevos en nidos de otras especies que se encargan del cuidado parental de sus crías, lo que disminuye, en consecuencia, el éxito reproductivo de la especie parasitada (Reboreda et al., 2003). De acuerdo a Lowther (2018) se han registrado 270 especies entre víctimas y hospedadoras de M. bonariensis, 97 de las cuales son hospedadoras. Según este autor, las hospedadoras son aquellas especies que muestran ser efectivas criando a los pichones parásitos. La razón por la cual el número de especies víctimas y hospedadoras de $M$. bonariensis es grande en comparación con otras especies de Molothrus puede ser su carácter parásito generalista.

Se registró en vídeo una relación de parasitismo entre un juvenil de $M$. bonariensis occidentalis y un Hornero Patipálido Furnarius leucopus cinnamomeus como hospedador (Fig. 1). Este registro se observó al sur de la ciudad de Guayaquil (-2,234253, -79,895022; 5 m s.n.m.), provincia de Guayas, Ecuador, en 24-25 de junio de 2020. El avistamiento ocurrió en ambos días en horas de la mañana, en dos parques que se encuentran separados c. $300 \mathrm{~m}$ entre sí. El primer día, un adulto $F$. leucopus se desplazaba forrajeando entre ambos parques y el juvenil M. bonariensis lo perseguía constantemente. Se observó que el adulto lo alimentó un par de veces. Ambos parques se encuentran en una zona urbana bastante ruidosa, pero esto no pareció afectar a que la insistente la cría sea alimentada por su hospedador. En el primer día, solo se observó a un individuo de $F$. leucopus con la cría, mientras que en el segundo día estaban presentes dos individuos, aunque el juvenil perseguía con insistencia apenas a uno de ellos, y solamente ese individuo lo alimentaba.

Las interacciones de parasitismo entre $M$. bonariensis y especies del género Furnarius son limitadas. Se ha reportado al Hornero Copetón $F$. cristatus como hospedero frecuente de este parasitismo (Hoy \& Ottow, 1964; Fraga, 1980; Mason \& Rothstein, 1986). Según Hoy \& Ottow (1964), F. rufus presenta un rechazo hacia los huevos de M. bonariensis, debido a que pueden diferenciarlos por su tamaño. Por su parte, Mason \& Rothstein (1986) sugieren que esta diferenciación varía de acuerdo a la distribución de $M$. bonariensis, ya que el tamaño de sus huevos varía geográficamente. El único registro publicado de parasitismo de M. bonariensis a F. leucopus fue en 2012 en Mindo, provincia de Pichincha, Ecuador (Medrano-Vizcaíno et al., 2020).

Wilson (1979) indica varios episodios de cuidado parental en parejas de $F$. rufus a juveniles de M. bonariensis en Argentina. Aunque solo observé a un individuo de F. leucopus atendiendo al juvenil M. bonariensis, no puedo descartar que el cuidado también haya sido biparental. El registro de la presente nota contribuye a comprender el parasitismo de puesta de M. bonariensis en Ecuador. La zona donde se registró esta interacción parásito-hospedador es una zona antrópica casi en su totalidad, como es recurrente en los eventos de parasitismo conocidos de esta especie (Medrano-Vizcaíno et al., 2020). 
Gracias a Stephanie Navas por su compañía en las observaciones; a Héctor Cadena por su motivación para escribir esta nota de campo; y a Rosendo Fraga y un revisor anónimo por sus sugerencias.

\section{REFERENCIAS}

Fraga, R. (1980). The breeding of the Rufous Hornero (Furnarius rufus). Condor, 82, 58-68. DOI: https://doi.org/10.2307/1366785

Hoy, G., \& Ottow, J. (1964). Biological and zoological studies of the molothrine cowbirds (Icteridae) of Argentina. The Auk, 81, 186-203. DOI: https://doi.org/10.2307/4082768

Lowther, P. E. (2018). Lists of victims and hosts of the parasitic cowbirds (Molothrus). Chicago, IL: The Field Museum. URL: https://www.fieldmuseum.org/sites/default/files/cowbird-hosts-05sep2018.pdf

Mason, P., \& Rothstein, S. (1986). Coevolution and avian brood parasitism: cowbird eggs show evolutionary response to host discrimination. Evolution, 40, 1207-1214. DOI: https://doi.org/10.2307/2408948

Medrano-Vizcaíno, P., Bedoya, J., Cadena-Ortiz, H. (2020). Dinámica de la distribución y hospederos de Molothrus bonariensis (Paseriformes: Icteridae) en Ecuador. Caldasia, 42(1), 38-49. DOI: https://doi.org/10.15446/caldasia.v42n1.78891

Reboreda, J. C., Mermoz, M. E., Massoni, V., Astié, A. A., \& Rabuffetti, F. L. (2003). Impacto del parastismo de cría del Tordo Renegrido (Molothrus bonariensis) sobre el éxito reproductivo de sus hospedadores. Hornero, 18(2), 77-88. URL: http://hdl.handle.net/20.500.12110/hornero v018 n02 p077

Wilson, D. B. (1979) Nota sobre casos de parasitismo del Renegrido (Molothrus bonariensis) sobre varios Passeriformes observados en la provincia de Corrientes. Hornero, 12(01): 69-71. URL: https://core.ac.uk/download/pdf/70305972.pdf

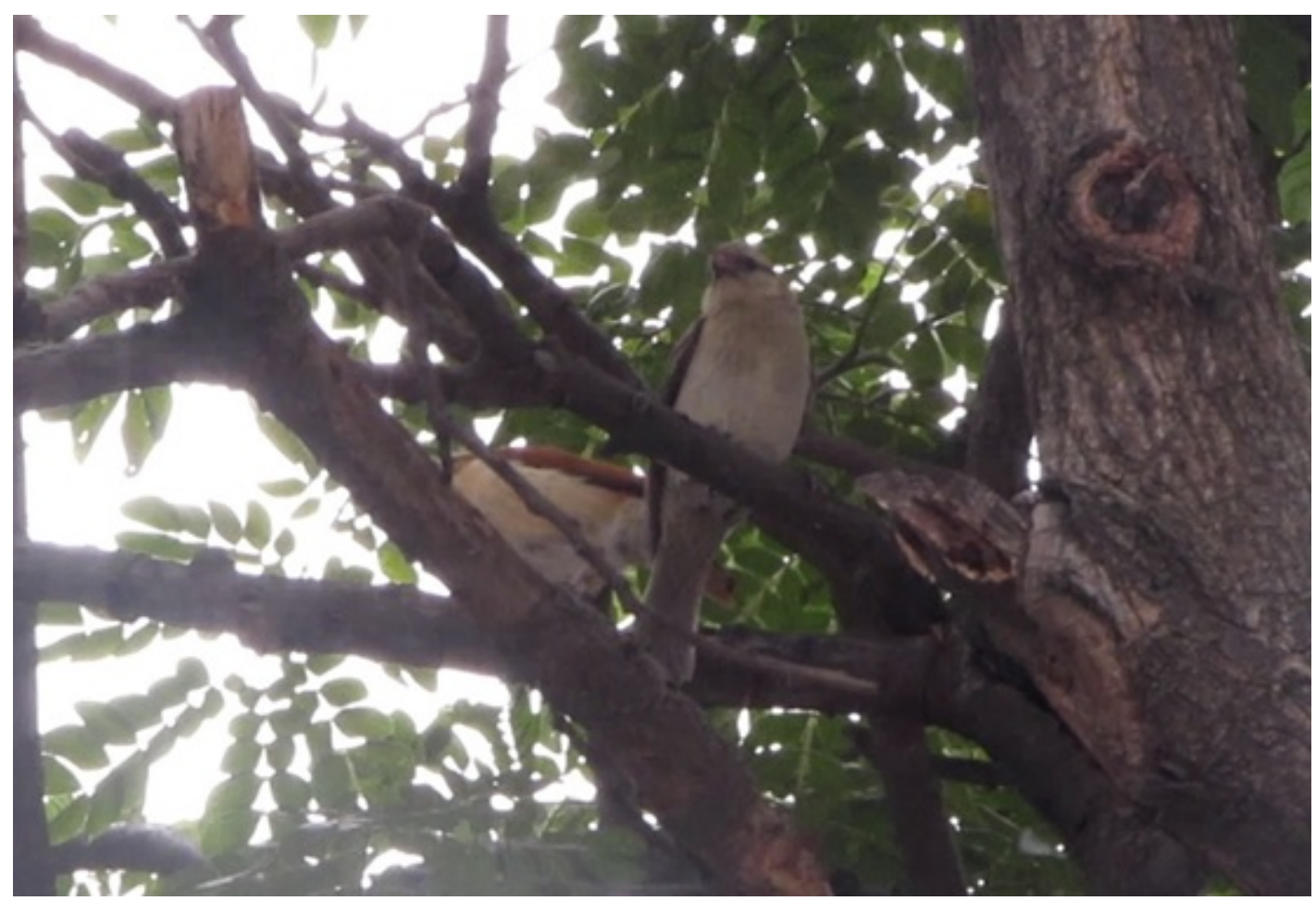

Figura 1: Juvenil de Vaquero Brilloso Molothrus bonariensis occidentalis perchado junto a adulto de Hornero Patipálido Furnarius leucopus cinnamomeus en Guayaquil, Guayas, 24 de junio de 2020 (Benjamín Navas). 